\title{
Une militante du parlementarisme européen: Simone Veil
}

\author{
Aurélie Élisa GFELLER
}

Simone Veil compte aujourd'hui parmi les figures les plus importantes de la vie publique française et européenne. Les rôles qu'elle a exercés sont multiples. Magistrate, elle fut l'une des rares femmes à siéger au Conseil constitutionnel, la plus haute instance administrative et juridictionnelle française. Ministre de la Santé en charge de la sécurité sociale sous Valéry Giscard d'Estaing, et inspiratrice de la loi du 17 janvier 1975 dépénalisant l'avortement, elle personnifie la lutte pour l'émancipation des femmes. Survivante de la shoah, elle évoque aussi le plus grand traumatisme que connut l'Europe au vingtième siècle. Mais c'est son engagement au sein du Parlement européen (PE) qui lui conféra une stature internationale. Elle y siégea pendant quatorze ans, du 17 juillet 1979 au 30 mars 1993, d'abord comme présidente du premier PE élu au suffrage universel direct jusqu'au 18 janvier 1982, puis comme simple députée et présidente du groupe libéral et démocratique de 1984 à $1989 .{ }^{1}$

Cet engagement européen surprit certains de ses proches. «Pourquoi est-elle devenue si européenne? Ses explications ne m'ont jamais tout à fait satisfaite», note Anne-Lise Stern, une amie revenue avec Veil d'Auschwitz. ${ }^{2}$ Veil affirme certes dans ses mémoires que dès les années cinquante, ni elle ni son mari «ne fais[aient] mystère de militer en faveur de la construction européenne». Selon elle, ils jugèrent tous deux que l'Assemblée nationale française avait commis une «lourde erreur» en refusant de ratifier en 1954 le traité instituant la Communauté européenne de défense (CED). ${ }^{3}$ Son parcours jusqu'en 1979 s'inscrivit cependant dans un cadre strictement national. Ce fut d'ailleurs Giscard qui lui proposa en 1978 de mener la liste UDF (Union pour la démocratie française) aux premières élections directes du PE. ${ }^{4}$ Non content du succès de la liste Veil, Giscard tint à porter cette ancienne déportée à la tête de l'assemblée comme symbole de la réconciliation franco-allemande. ${ }^{5}$ Il œuvra dans ce sens auprès du chancelier ouest-allemand, Helmut Schmidt. ${ }^{6}$ Son ministre des Affaires étrangères, Jean François-Poncet, fut chargé de persuader ses collègues européens, tandis que des centristes nouvellement élus au PE - Michel Poniatowski, ancien ministre, ou Jean Lecanuet - agirent en coulisse à Strasbourg. L'élection de Veil résulta, semble-t-il, d'un savant compromis: on s'accorda pour donner la présidence du groupe libéral à un élu allemand, celle de la commission agricole à un conservateur britannique, et celle de la commission politique à un démocrate-chrétien italien. Veil

1. PARLEMENT EUROPÉEN, Vos députés: Simone Veil [cité 26.08.2010]; disponible sur http:// www.europarl.europa.eu/members/archive/alphaOrder/view.do;jsessionid=59B0A475F61B427A3234998E829E34BD.node2 ?language=FR\&id=1174.

2. M. SZAFRAN, Simone Veil. Destin, Éd. Flammarion, Paris, 1994, p.331. Sur Stern, voir ibid., p.232.

3. S. VEIL, Une vie, Éd. Stock, Paris, 2007.

4. Ibid., p.179.

5. Ibid., p.181.

6. Sur les dessous de l'élection de Veil à la présidence, voir M. SZAFRAN, op.cit., pp.328-330. 
s'imposa très vite ensuite comme une militante de la cause européenne. Elle ne fit certainement pas partie de ces députés qui considéraient le PE comme un pis-aller qu'il s'agisse d'une retraite forcée, temporaire ou définitive, de l'arène politique nationale ou d'un point d'entrée dans celle-ci. ${ }^{7}$ La longévité de son mandat et son activisme - elle intervint deux cent cinquante fois en séance plénière - témoignent d'un réel engagement. ${ }^{8}$

La trajectoire de Veil soulève ainsi la question des pouvoirs de socialisation et plus particulièrement d'européanisation du PE. L'appartenance à une institution parlementaire transnationale tendait-elle à modifier dans un sens pro-européen la vision d'hommes et de femmes issus de la scène politique nationale? Question épineuse, s'il en est, à laquelle se sont attaqués les politologues. Les résultats de leurs questionnaires, entretiens et analyses statistiques à variables multiples suggèrent une réponse négative. ${ }^{9}$ Les institutions européennes, concluent-ils, ne contribuent guère à européaniser leurs membres. Un profil pro-européen plus marqué serait plutôt le résultat d'un effet d'auto-sélection. Cette explication ne vaut pas pour Veil, puisqu'elle ne s'engagea pas publiquement au service de la cause européenne avant les élections de 1979. Pour autant, il est peu vraisemblable que le PE ait transformé son profil à travers un processus de socialisation institutionnelle, comme le postulent les études sur l'européanisation. ${ }^{10}$ Dans la mesure où Veil assuma aussitôt la présidence du PE, elle ne fut pas soumise aux mêmes mécanismes de socialisation qu'une nouvelle recrue relativement peu expérimentée dans le cadre, par exemple, d'une commission parlementaire. Il faut donc plutôt voir dans son militantisme le reflet de la conception qu'elle se faisait de son rôle.

Cet article analyse ainsi le parcours européen de Veil sur la base de la typologie des rôles et des orientations des parlementaires européens établie par les politologues

7. Sur les usages du PE et sa place dans les carrières politiques nationales, voir N. KAUPPI, La construction de l'Europe : le cas des élections européennes finlandaises en 1999, in: Cultures \& conflits, 38-39(1999), pp.101-118; N. KAUPPI, European Union Institutions and French Political Careers, in: Scandinavian Political Studies, 1(1996), pp.1-24. W. BEAUWALLET, Institutionnalisation et professionnalisation de l'Europe politique. Le cas des eurodéputés français, in: Politique européenne, 9(2003); G. MARREL, R. PAYRE, Des carrières au Parlement. Longévité des eurodéputés et institutionnalisation de l'arène parlementaire, in: Politique européenne, 1(2006), pp. 69-104.

8. Base de données du Centre archivistique et documentaire (CARDOC) du Parlement européen (PE) à Luxembourg.

9. M.N. FRANKLIN, S.E. SCARROW, Making European? The Socializing Power of the European Parliament, in: R.S. KATZ, B. WESSELS (éds.), The European Parliament, National Parliaments, and European Integration, Oxford University Press, Oxford, 1999; L. HOOGHE, Several Roads Lead to International Norms, but Few via International Socialization: A Case Study of the European Commission, in: International Organization, 4(2005), pp.861-898; R. SCULLY, Becoming Europeans? Attitudes, Behaviour, and Socialization in the European Parliament, Oxford University Press, Oxford/New York, 2005.

10. Voir, par exemple, R.F. FENNO, Congressmen in Committees, Little Brown, Boston, 1973; R. PUTMAN, The Beliefs of Politicians: Ideology, Conflict, and Democracy in Britain and Italy, Yale University Press, New Haven, 1973. 
Tim Bale et Paul Taggart. ${ }^{11}$ Bale et Taggart distinguent quatre «types-idéaux»: le militant (policy advocate), le représentant de circonscription, l'évangéliste européen et l'institutionnaliste. Ils présentent ces catégories comme mutuellement exclusives même s'ils admettent que l'orientation d'un député puisse changer au cours du temps. ${ }^{12}$ À l'instar d'autres politologues usant de ce cadre conceptuel, nous estimons au contraire qu'un parlementaire pouvait assumer simultanément plusieurs rôles. ${ }^{13}$ Au début de son mandat européen, Veil endossa essentiellement le rôle d' «évangéliste européenne». Au fil du temps, elle assuma également celui de «militante» (policy advocate), tandis qu'elle s'investissait sur des dossiers tels que les droits de l'homme et la santé publique - thèmes qu'elle contribua à promouvoir sur la scène européenne.

\section{Une militante de l'Europe unie}

Veil s'engagea au service de la cause européenne dès son arrivée au PE. Une Europe unie lui apparaît essentielle pour préserver non seulement la diversité des cultures européennes ${ }^{14}$ mais aussi un modèle de société qui allie l'initiative et la responsabilité individuelles à «la prise en charge par la collectivité de certains besoins essentiels». ${ }^{15}$ Cette vision s'inscrit dans un courant de pensée libérale. Sa conception du libéralisme n'était cependant pas celle des tenants du néo-libéralisme économique en vogue outre-Manche et outre-Atlantique depuis l'élection de Margaret Thatcher et de Ronald Reagan. Plaidant pour un juste milieu entre laissez-faire et interventionnisme étatique, ${ }^{16}$ elle prône un libéralisme «humaniste» - un humanisme qui repose sur les principes de pluralisme, liberté d'expression et libre circulation des idées et des individus. ${ }^{17} \mathrm{C}$ 'est précisément ce principe de libre flux des échanges, soulignet-elle, qui inspira la création du marché commun. Qu'il s'agisse ou non d'une justi-

11. T. BALE, P. TAGGART, The Newest of the New? Accession State MEPs: Who they are and Who They Think They Are, communication présentée à l'occasion du Federal Trust Workshop «The European Parliament and the European Political Space», mars 2006, Londres, p.7. Version électronique: http://www.eu-consent.net/library/team08/Bale-Taggart060330.pdf [dernière date d'accès: 29 janvier 2011]. Cette typologie nous parait plus utile pour la période considérée dans cet article que les catégories proposées par Richard Katz - soit celles d'agent (catégorie qui correspond au représentant de circonscription de Bale et Taggart), d'administrateur (trustee) et de tenant d'une Europe fédérale ou intergouvernementale: R.S. KATZ, Representational Roles, in: European Journal of Political Research, 32(1997), pp.211-226.

12. T. BALE, P. TAGGART, op.cit., p.8.

13. R.S. KATZ, op.cit, p.217.

14. PE1 P1 102/PARR PARR-19801217 0070, Veil, Discours prononcé devant le Collège d'Europe à Bruges, 01.10.1980.

15. PE1 P1 102/PARR PARR-19801217 0105, Veil, Discours prononcé à l'occasion de la 4e conférence Jean Monnet, 27.11.1980.

16. PE1 P1 102/PARR PARR-19830904 0100, Veil, Discours: «L'Europe des libertés» prononcé lors des dîners-rencontres versaillais, 05.12.1983; PE1 P1 102/PARR PARR-19840118 0100, Discours prononcé lors des journées parlementaires de l'UDF, 25.09.1984.

17. Discours prononcé lors des journées parlementaires de l'UDF, 25.09.1984, op.cit. 
fication a posteriori de son engagement, une telle affirmation montre combien Veil s'identifie désormais au projet européen. Certes, à défaut de lettres ou de procèsverbaux d'entretiens, l'historien doit s'en remettre à ses discours. ${ }^{18}$ Veil remaniait cependant les ébauches de ses collaborateurs. ${ }^{19}$ De plus, lorsqu'elle redevint simple députée, elle écrivit sans doute elle-même ses textes, du moins en partie. ${ }^{20}$

Autre signe du rôle d'évangéliste européenne qu'endosse Veil, sa vision fédérale d'une Europe unie. Ce n'est qu'au fil du temps qu'elle constatera «un attachement croissant des citoyens à leur cadre national» et qu'elle en viendra donc à concevoir l'Union européenne comme «un agrégat de poupées russes». ${ }^{21} \mathrm{Au}$ début des années quatre-vingt elle imagine encore «une évolution vers un système de type fédéral». ${ }^{22}$ Les citoyens européens, déclare-t-elle en décembre 1981, sont de plus en plus conscients du fait que «l'Europe peut et doit avoir une réalité et un rôle propres à coté de ceux que peuvent avoir les nations individuellement». ${ }^{23}$ Il est donc logique qu'elle approuve en 1984 le Projet de traité pour l'Union européenne. ${ }^{24}$ Ce projet, rédigé sous l'impulsion du député fédéraliste italien Altiero Spinelli, plaidait non seulement pour la réalisation du marché intérieur mais aussi pour des réformes d'envergure visant à renforcer les institutions communautaires. Surtout, elle n'a de cesse de défendre les prérogatives du PE, institution susceptible de se muer en organe de décision démocratique d'une fédération européenne. Elle met en avant sa nouvelle légitimité démocratique depuis les élections directes de 1979 et le présente même comme «la force politique réellement européenne nécessaire pour faire progresser l'Europe». ${ }^{25} \mathrm{~L}$ 'engagement de Veil va au-delà des mots. En décembre 1979, elle soutient une majorité d'élus dans leur refus d'approuver le projet de budget soumis par le Conseil des

18. Les archives du cabinet Veil au PE ne contiennent aucun procès-verbal d'entretiens. Elles comportent en revanche une série qui regroupe les allocutions qu'elle prononça entre 1979 et 1984.

19. Voir, par exemple, PE1 P1 102/PARR PARR-19801217 0070, Schéma discours Bruges (annoté), 12.09.1980; Allocution de Madame Simone Veil devant le Collège d'Europe à Bruges le 1er octobre 1980 (texte annoté).

20. Veil opta pour une structure de travail légère: un bureau à son domicile parisien avec seulement une, puis deux assistantes (M. SARAZIN, Une femme, Simone Veil, Robert Laffont, Paris, 1987, pp. 240-242).

21. S. VEIL, Une vie, op.cit., pp.189-190.

22. S. VEIL, Discours: "Le Parlement européen: bilan d'une législature», in: Revue du marché commun, 275(1984), p.189.

23. PE1 P1 215/VISI VISI-19811203 0120, Veil, Discours devant les membres de l'Assemblée du peuple de la République arabe d'Egypte, 05.12.1981.

24. Projet de traité instituant l'Union européenne, in: Bulletin des Communautés européennes, 2(1984), pp.8-26. PE1 P1 102/PARR PARR-19840118 0020, Veil, Discours au Parlement européen sur «L'Union européenne», 14.02.1984.

25. S. VEIL, Discours: «Le Parlement européen: bilan d'une législature», op.cit., p.189. Voir aussi PE1 P1 102/PARR PARR-19840118 0100, Veil, Discours prononcé lors des journées parlementaires de l'UDF, 25.09.1984 et Discours devant les membres de l'Assemblée du peuple de la République arabe d'Egypte. 
ministres des Finances - un droit qu'avait conféré au PE le traité du 22 juillet 1975. ${ }^{26}$ Le Premier ministre français, Raymond Barre, pour lequel elle avait travaillé comme ministre, ne se fit pas faute d'exercer des pressions. Il eut beau téléphoner «vingt fois», alerter la presse sur la «"trahison" de Veil à l'égard de la France» et «élever le ton pour [la] rappeler à [s]es "devoirs envers [s]on pays"», celle-ci ne céda pas. ${ }^{27} \mathrm{Il}$ y avait des enjeux de fonds. Les élus souhaitaient mettre un frein aux dépenses agricoles et promouvoir les politiques d'aide extérieure. ${ }^{28}$ Mais au-delà de tels désaccords de fonds, le PE et sa présidente avaient clairement engagé une épreuve de force avec le Conseil.

\section{Promouvoir la voix de l'Europe sur la scène internationale}

Veil témoigne aussi de son évangélisme européen dans les efforts qu'elle fait pour accroître la visibilité internationale de l'Europe. Elle prend ainsi l'initiative d'inviter au PE Anouar el-Sadate, président égyptien et Prix Nobel de la paix. ${ }^{29}$ Le retentissement du discours de Sadate en 1981 fut tel qu'à Paris, où le dirigeant égyptien devait poursuivre sa visite, on en prit ombrage. ${ }^{30}$ Sa présence à Strasbourg avait valeur de symbole. Veil ne se fit pas faute de le souligner. Cette visite, déclara-t-elle, signalait la part qu'il souhaitait voir prendre par l'Europe dans le processus de paix au MoyenOrient. ${ }^{31}$ Il ne s'agissait pas d'un vœu pieux. Le conflit israélo-arabe avait été l'un des thèmes majeurs de la Coopération politique européenne (CPE) dès sa création en 1970 - un processus qui avait débouché sur le lancement par le Conseil européen de Venise en 1980 d'une initiative sur le Moyen-Orient. ${ }^{32}$

C'est aussi par ses voyages que Veil tente de faire porter la voix de l'Europe. Au cours de sa présidence, elle sillonne les pays candidats à l'adhésion, les États-Unis,

26. P. GERBET, Parlement européen, in: P. GERBET, G. BOSSUAT, T. GROSBOIS (éds.), Dictionnaire historique de l'Europe unie, André Versaille éd., Paris, 2009, pp.742-754; B. RITTBERGER, The Creation and Empowerment of the European Parliament, in: Journal of Common Market Studies, 2(2003), p.217.

27. S. VEIL, Une vie, op.cit., p.187.

28. PE1 P1 222/BUDG BUDG-19791012 0020, PE, Résolution sur le projet de budget général des Communautés pour l'exercice 1980, 07.11.1979.

29. S. VEIL, Une vie, op.cit., p.224.

30. PE1 P1 214/RECE RECE-19810209 0190, PE, Note manuscrite relative à la présence de la presse, sans date.

31. PE1 P1 214/RECE RECE-19810209 0130, Veil, Discours prononcé à l'occasion de la visite de Anouar el Sadate, séance solennelle, 10.02.1981.

32. Statement by the European Council on the Situation in the Middle East (Venice Declaration), Venice, 12-13 June 1980, in: C. HILL, K.E. SMITH (éds.), European Foreign Policy Key Documents, Routledge, London/New York, 2000, pp.302-304. Sur la CPE au Moyen-Orient, voir A.E. GFELLER, A European Voice in the Arab World: France, the Superpowers and the Middle East, 1970-1974, in: Cold War History, (forthcoming); D. MÖCKLI, European Foreign Policy During the Cold War: Heath, Brandt, Pompidou and the Dream of Political Unity, I.B. Tauris, London, 2009, pp.184-208. 
le Moyen-Orient ou encore la Chine. Ses visites au Portugal et en Grèce visent à renforcer les liens entre le PE et le parlement national avant l'adhésion. ${ }^{33}$ Son voyage en Chine s'inscrit dans une perspective politico-économique:

«La Chine, grande puissance mondiale et pays à commerce d'État, a une très grande importance politique, et [...] elle est susceptible de donner une ampleur considérable au développement des échanges». ${ }^{34}$

Les États-Unis, insiste-t-elle, constituent le premier partenaire économique et commercial de la Communauté. ${ }^{35}$ Ils assurent aussi sa sécurité. Veil ne fait pas partie de ceux qui prônent un relâchement des liens transatlantiques. Bien au contraire, elle juge que tant qu'il n'y aura pas de réelle défense européenne, l'Europe se doit d'être solidement ancrée dans l'Organisation du Traité de l'Atlantique Nord (OTAN). ${ }^{36}$ Ses critiques n'épargnent donc pas les leaders politiques - pour la plupart socio-démocrates, verts et communistes - qui condamnent l'installation par l'OTAN de missiles Pershing en réponse au déploiement de missiles SS 20 par l'Union soviétique. ${ }^{37}$

Veil reprend le fil de ses voyages à la suite de son élection à la présidence du groupe libéral en 1984. Favorable au principe d'élargissement, elle se rend à plusieurs reprises à Madrid, Barcelone et Lisbonne. Ces contacts s'étendent ensuite au-delà du continent européen, particulièrement à l'Amérique latine, «très courtisée $[\ldots]$ par les libéraux allemands», soucieux de promouvoir les intérêts économiques de leur pays. ${ }^{38}$ C'est cependant sur la situation politique du pays qu'insiste Veil à son retour d'un voyage au Chili en 1985. Ce séjour, déclare-t-elle, lui a montré qu'il existait «une véritable alternative [...] démocratique» sous l'autorité morale du cardinal Juan Francisco Fresno. ${ }^{39}$ Elle regrette donc que le général Augusto Pinochet n'ait su répondre à l'attente populaire d'un accord national que par la violence.

\section{Une tribune pour dénoncer les atteintes aux droits de l'homme}

Au fil du temps, Veil s'engage également sur d'autres thèmes, assumant ainsi le rôle de «militante» (policy advocate). Les droits de l'homme figurent en première ligne.

33. Sur la Grèce, voir PE1 P1 215/VISI VISI-19800328 0080, Bureau du PE, extrait du procès-verbal de réunion, 10.09.1979; sur le Portugal, voir PE1 P1 215/VISI VISI-19810517 0070, Leonardo de Ribeira, président du Portugal, à Veil, 23.03.1981.

34. PE1 P1 215/VISI VISI-19810717 0060, PE, Note relative au rôle du Parlement européen dans les relations entre la CEE et la Chine, sans date.

35. PE1 P1 215/VISI VISI-19800128 0060, Veil, Discours devant le National Press Club de Washington, 28.01.1980.

36. Veil, Discours : «L'Europe des libertés», 05.12.1983, op.cit.

37. T. JUDT, Postwar: A History of Europe Since 1945, Penguin Press, New York, 2005, pp.590-591.

38. S. VEIL, Une vie, op.cit., p.203 et 204.

39. PE2 AP DE/1985 DE19850912-02 0010, Veil, Intervention orale en séance plénière du PE, 12.09.1985. 
Présidente du PE, Veil use de son statut pour plaider en faveur de la libération de détenus politiques. ${ }^{40}$ Devenue simple députée, elle exhorte «l'Europe» à

«encourager le développement des jeunes nations qui se sont lancées dans la difficile aventure de la démocratie, et à défendre, partout où elles [sic] sont menacés les droits et la dignité de la personne humaine». ${ }^{41}$

Le PE, estime-t-elle, a un rôle important à jouer par ses prises de position. ${ }^{42} \mathrm{Il}$ s'agit pour lui de «dénoncer sans cesse, toutes les atteintes aux droits de l'homme». Même si les gouvernements des États membres ne peuvent tenir compte de ses résolutions, ajoute-t-elle, «nous sommes en effet un Parlement et c'est comme cela que nous serons crédibles». ${ }^{43}$ Joignant le geste à la parole, elle s'exprimera à de multiples reprises sur ce thème. Ainsi, en 1983, elle prononce un vibrant plaidoyer en faveur des juifs d'Union soviétique qui étaient soumis à des discriminations et se voyaient refuser tout visa de sortie. ${ }^{44}$ Autre signe de son engagement, 22 de ses 250 interventions en séance plénière traitent des droits de l'homme stricto sensu, tout comme 4 des 30 questions qu'elle signa ou cosigna. ${ }^{45} \mathrm{C}$ 'est sans compter les fois où elle aborda ce sujet dans le cadre d'un débat portant sur un pays en particulier comme la Roumanie en $1989 .{ }^{46}$

Les interventions de Veil témoignent d'une conscience aiguë du conflit potentiel entre droits humains et intérêts politico-économiques:

Sommes-nous prêts à suspendre des relations commerciales à un moment où nous avons autant besoin de nos partenaires? Sommes-nous prêts à suspendre des livraisons d'armes, quitte à fermer des entreprises? Sommes-nous prêts à faire la guerre pour la Pologne? Sommes-nous prêts à quitter les organisations internationales dont on reconnaît qu'elles utilisent leur programme à des fins politiques? Non! Nous sommes en fait condamnés à vivre ensemble. ${ }^{47}$

Pour autant, elle ne prône pas une soumission aux diktats de la «Realpolitik». À la suite de l'assassinat en 1991 de Chapour Bakthiar, ancien Premier ministre du shah refugié en France, elle engage ainsi les démocraties à juger les assassins, quels que

40. PE1 P1 105/DROI DROI-19790715 0350, Veil à Jorge Rafael, président de la République argentine, 17.10.1980; PE1 P1 105/DROI DROI-19800522 0020, Veil à Cenpa Keskin, ambassadeur turc auprès de la Communauté, 23.12.1981; PE1 P1 105/DROI DROI-19800522 0180, PE, Note: interventions concernant les droits de l'homme faites par le président auprès des autorités turques, 13.01.1982.

41. Veil, Discours : «L'Europe des libertés», 05.12.1983, op.cit.

42. Veil, Discours devant le Collège d'Europe à Bruges, 01.10.1980, op.cit.

43. PE1 P1 102/PARR PARR-19830105 0070, Veil, Discours prononcé dans le cadre du débat en séance plénière sur les droits de l'homme, 18.05.1983.

44. PE1 P1 102/PARR PARR-19830105 0050, Veil, Discours prononcé devant le Comité de soutien des Juifs d'URSS, 14.03.1983.

45. CARDOC, base de données.

46. Résolution sur la Roumanie (16.04.1989), in: Journal officiel des Communautés européennes (dorénavant: JO), C96(1989).

47. Veil, Discours prononcé dans le cadre du débat sur les droits de l'homme, 18.05.1983, op.cit. 
soient leurs rapports avec les États dont ils sont issus. ${ }^{48}$ Naturellement, Veil n'agit pas seule. En septembre 1991, une majorité d'élus vote une résolution qui met en cause la responsabilité du gouvernement iranien dans cet assassinat et déplore que «certains États membres, à la recherche d'avantages diplomatiques ou commerciaux, semblent vouloir ignorer les actions criminelles» de l'Iran. ${ }^{49}$ Mais Veil se distingue par son franc-parler. S'exprimant en faveur des juifs d'Union soviétique en terre israélienne, elle n'hésite pas à marquer ses inquiétudes sur le sort des populations arabes. Dès lors que les règles légales ne suffisent plus pour ramener le calme, déclaret-elle même, «le respect des droits de l'homme est menacé». ${ }^{50}$ Lorsqu'éclate la guerre civile en Bosnie, elle fustige l'hypocrisie des dirigeants occidentaux qui dénoncent les exactions perpétrées dans les camps de détention sans pour autant se montrer prêts à accueillir les victimes. «Nous ne pouvons pas tolérer qu'il y ait un double langage», martèle-t-elle..$^{51}$ Elle va jusqu'à attaquer ses collègues parlementaires pour leur refus d'étendre à la Croatie un programme d'aide communautaire aux pays d'Europe centrale et orientale (PHARE) - un programme qui aurait permis, estime-t-elle, d'améliorer le sort de nombreuses femmes réfugiées. ${ }^{52}$ «On ne peut pas demander aux autres institutions de faire quelque chose quand nous-mêmes nous refusons de le faire», assène-t-elle, qualifiant d' «hypocrisie scandaleuse» l'attitude du PE. ${ }^{53}$

\section{Pour une politique européenne de la santé}

Autre thème clé sur lequel s'engage Veil au PE: la santé. Il n'y là rien qui doive surprendre, compte tenu des fonctions qu'elle avait exercées comme ministre de la Santé. Dès 1980, elle s'exprime en faveur d'un grand débat parlementaire européen sur la question. Dans un discours prononcé à Luxembourg en juillet 1980, elle déclare qu'«une réflexion communautaire est propre à apporter des idées novatrices ayant des retombées sociales et économiques importantes pour les citoyens de l'Europe». ${ }^{54}$ Ses vœux finiront pas être exaucés. En 1983, la Commission européenne annonce l'élaboration d'une directive sur la «formation spécifique du médecin gé-

48. PE3 AP DE/1991 DE19910912-04 0160, Veil, Intervention en séance plénière du PE, 12 septembre 1991.

49. I. ERICH, Une avancée décisive de l'enquête. L'assassinat de Chapour Bakhtiar aurait été commandité de Téhéran, in: Le Monde, 2109 1991; Résolution sur l'assassinat de M. Chapour Bakthiar (12.09.1991), in: JO, C267(1991).

50. Veil, Discours prononcé devant le Comité de soutien des Juifs d'URSS, 14.03.1983, op.cit.

51. PE3 AP DE/1992 DE19921029-08 0060, Veil, Intervention en séance plénière du PE, 29.10.1992.

52. COMMISSION EUROPÉENNE, Europa. Synthèses de la législation de l'UE [cité 25.08.2010]); disponible sur http://europa.eu/legislation_summaries/enlargement/2004_and_2007_enlargement/ e50004 fr.htm.

53. PE3 AP DE/1993 DE19930311-01 0310, Veil, Intervention en séance plénière du PE, 11.03.1993.

54. PE1 P1 102/PARR PARR-19801217 0030, Veil, Discours prononcé à l'occasion du Symposium international sur le «Rôle du médecin en éducation pour la santé» (texte reproduit d'après la bande d'enregistrement), 02.07.1980. 
néraliste». La proposition tarde. Veil s'impatiente, comme en atteste sa question en date du 10 octobre 1984. ${ }^{55} \mathrm{La}$ Commission se fait fort de la rassurer: une proposition de directive sera promptement transmise au Conseil des ministres. ${ }^{56}$ C'est sur ce projet de directive que Veil rédigera un rapport pour avis. ${ }^{57}$ En séance plénière, elle félicitera la Commission pour son œuvre de concertation avec la profession médicale et pour les mesures proposées afin de freiner l'augmentation des coûts - une problématique relativement nouvelle qui l'avait déjà occupée durant son mandat ministériel. ${ }^{58}$

Veil consacre un autre rapport à l'un de ses chevaux de bataille: la prévention du cancer et la lutte contre le tabagisme. C'est sous son impulsion que la France avait lancé une politique de lutte contre le tabagisme. Donnant l'exemple, Veil avait cessé de fumer en public dès $1975 .{ }^{59} \mathrm{La}$ loi du 9 juillet 1976 réglementa ensuite la publicité et introduisit une interdiction de fumer dans certains lieux publics et milieux confinés. Bien qu'inspirées des recommandations de l'Organisation mondiale de la santé, ces dispositions exposèrent Veil aux critiques. On la traita même de «névrosée». ${ }^{60}$ Les progrès de l'action communautaire lui permirent de revenir à la charge au PE. En 1985, les Conseils européens de Milan et de Luxembourg décidèrent en effet de promouvoir la lutte contre le cancer. Trois ans plus tard, le PE fut chargé d'examiner une série de propositions. En séance plénière, Veil rendit hommage aux auteurs du rapport parlementaire sur la question pour avoir su résister aux pressions des lobbies du tabac. ${ }^{61}$ Elle fustigea également les amendements visant à réduire les crédits affectés aux campagnes d'information sous prétexte qu'elles étaient inutiles. En 1990, c'est à son tour de produire un rapport sur un plan d'action contre le cancer pour 1990-1994. ${ }^{62}$ Elle se montre très critique face aux mesures suggérées par la Commission - des campagnes d'information, des expériences pilotes et une évaluation des programmes en cours - qu'elle juge tout à fait insuffisantes. Elle préconise une approche ambitieuse qui ouvre la voie à «une véritable politique européenne de santé publique». ${ }^{63}$ Une telle approche implique une politique de dépistage systématique, mais aussi une vision globale du patient dans ses souffrances physiques et psychiques, avec la création de centres de soins palliatifs. Mais c'est la proposition d'interdire

55. PE2 AP QP/QH H-0337/84 0020, Veil, Question orale H-337/84, 10.10.1984.

56. $\mathrm{PE} 2 \mathrm{AP} \mathrm{QP} / \mathrm{QH} \mathrm{H}-0337 / 84$ 0030, [Commission européenne], Réponse à la question orale H-337/84, sans date.

57. PE2 AP RP/JURI.1984 A2-0150/85 0010, Veil, Avis de la commission de l'environnement, de la santé publique et de la protection des consommateurs, 19.09.1985,.

58. PE2 AP DE/1985 DE19860115-04 0020, Veil, Intervention en séance plénière du PE, 15.01.1986. Voir aussi M. SARAZIN, Une femme, ..., op.cit., pp.182-186.

59. Ibid., p.187.

60. PE2 AP DE/1986 DE19860512-03 0110, Veil, Intervention en séance plénière du PE, 12.05.1986.

61. PE2 AP DE/1987 DE19880211-07 0070, Veil, Intervention en séance plénière du PE, 11.02.1988.

62. PE3 AP RP/ENVI.1989 A3-0052/90 0010, Veil, Rapport fait au nom de la commission de l'environnement, de la santé publique et de la protection des consommateurs sur la Proposition de la Commission au Conseil relative à une résolution concernant un plan d'action 1990-1994 dans le cadre du programme «L'Europe contre le cancer» (COM/89/210 - C3-83/89), 27.02.1990.

63. PE3 AP DE/1990 DE19900314-04 0070, Veil, Intervention en séance plénière du PE, 14.03.1990. 
toute publicité pour le tabac qui constitue la principale pierre d'achoppement. ${ }^{64}$ Vasso Papandreou, la commissaire en charge du dossier, déclare d'emblée que la Commission n'est pas encore en mesure d'accepter une telle disposition. Les élus se trouvent donc dans une impasse. S'ils votent cet amendement, celui-ci ne pourra déboucher sur aucune législation. ${ }^{65}$ Ils l'approuveront néanmoins, exigeant ainsi des «actions visant à ce que toute publicité concernant le tabac soit totalement interdite dans tous les États membres de la Communauté et ce, dans les plus brefs délais». ${ }^{66}$

La ténacité n'est pas une qualité qui fait défaut à Veil. Elle s'engage à nouveau sur cette question lorsque le PE examine en 1992 une proposition de directive en matière de publicité pour le tabac. ${ }^{67}$ Contrant l'argumentaire de la démocrate-chrétienne allemande Ursula Schleicher, elle se prononce en faveur de «certaines atteintes» à l'économie de marché dès lors qu'il s'agit de la santé.

Mais de quelle économie de marché, Madame Schleicher? Celle qui conduit à la mort? C'est cette économie de marché-là que vous préconisez? Moi je crois à l'économie de marché, mais pas à celle qui se permet de faire n'importe quoi et qui ferme les yeux sur la mort. ${ }^{68}$

Sa verve lui vaudra les critiques de la démocrate-chrétienne luxembourgeoise Astrid Lulling. Veil déplorera le caractère personnalisé du débat, mais elle se reconnaîtra volontiers «passionnée» dès lors que la santé des jeunes est en jeu. ${ }^{69}$ Une majorité d'élus votera d'ailleurs en faveur des amendements destinés à rendre la directive plus contraignante. ${ }^{70}$

\section{Conclusion}

Veil entra au PE dotée d'un fort «capital symbolique», pour reprendre la terminologie de Pierre Bourdieu. ${ }^{71}$ Nul besoin pour elle de se servir du PE pour faciliter une carrière

64. PE3 AP DE/1990 DE19900313-08 0030, Veil, Intervention en séance plénière du PE, 13.03.1990.

65. PE3 AP DE/1990 DE19900314-08 0080, Veil, Intervention en séance plénière du PE, 14.03.1990.

66. Plan d'action contre le cancer - proposition de résolution COM(89) 210 final. Proposition de résolution du Conseil concernant un plan d'action 1990-1994 dans le cadre du programme «l'Europe contre le cancer» approuvée avec les modifications suivantes (14 03 1990), in: JO, C96(1990), pp.72-80. Plan d'action contre le cancer (vote), in: JO, C96(1990), pp.45-46.

67. PE3 AP DE/1991 DE19920116-04 0120, Veil, Intervention en séance plénière du PE, 16.01.1992; PE3 AP DE/1991 DE19920116-04 0280, Veil, Intervention en séance plénière du PE, 16.01.1992; PE3 AP DE/1991 DE19920211-05 0040, Veil, Intervention en séance plénière du PE, 11.02.1992.

68. PE3 AP DE/1991 DE19920116-04 0120, Veil, Intervention en séance plénière du PE, 16.01.1992.

69. Ibid.

70. Proposition modifiée de directive du Conseil en matière de publicité en faveur des produits du tabac, in: JO, C67(1992), pp.35-38.

71. W. BEAUWALLET, S. MICHON, Les femmes élues au Parlement européen (1979-1984): modes de recrutement et stratégies d'investissement à l'Europe, in: Regards sociologiques, 27-28(2004), p.82. 
politique nationale qu'elle n'avait d'ailleurs pas véritablement recherchée. ${ }^{72} \mathrm{Ce}$ capital symbolique lui permit non seulement d'être élue à la présidence du PE sans avoir jamais siégé au sein de l'assemblée, mais aussi d'agir sans avoir à faire ses preuves. Elle ne suivit donc pas la trajectoire de nombreuses élues qui tentaient de compenser la faiblesse de leur position initiale - une position souvent périphérique dans l'arène nationale - par un fort investissement dans les travaux des commissions. ${ }^{73}$ Veil fit certes partie de plusieurs d'entre elles - notamment la commission juridique, dont elle assuma la présidence entre 1982 et 1984, et la commission de l'environnement, de la santé publique et de la protection des consommateurs de 1984 à 1986 et de 1989 à $1992 .{ }^{74}$ Mais elle ne s'investit pas beaucoup dans leurs activités. Trois rapports pour avis et deux rapports principaux, c'est en effet bien peu pour un mandat parlementaire qui dura, si l'on exclut ses deux ans et demi de présidence, plus de douze ans. ${ }^{75}$ Elle n'était pas pour autant passive ou indifférente, comme en témoignent ses multiples interventions en séance plénière et la longévité de son engagement. Mais elle agit essentiellement seule ou dans le cadre du groupe libéral par des interventions, des projets de résolution et des questions aux autres instances communautaires.

Pour autant, le parcours de Veil nous éclaire sur certaines des facettes de ce parlementarisme européen naissant. L'importance du verbe, qui ressort de ses discours, interventions et projets de résolution, est significative. Avant l'introduction de la procédure de co-décision par le traité de Maastricht en 1992 et l'extension de son champ d'application par les traités d'Amsterdam (1999) et de Lisbonne (2007), le PE jouissait uniquement de pouvoirs budgétaires limités. Il était donc logique qu'il situe essentiellement son action sur un plan déclaratoire. C'est ce qui explique l'insistance de Veil sur l'importance pour le PE de dénoncer toute atteinte aux droits humains et à la démocratie.

La controverse budgétaire de 1979-1980 illustre la stratégie du PE pour modifier l'équilibre inter-institutionnel communautaire. Qu'il s'agisse du rejet du projet de budget - opération répétée en 1982 - ou d'un dépassement du taux d'augmentation autorisé, les élus usaient de leurs prérogatives budgétaires pour renforcer leur position et celle de leur institution. ${ }^{76}$ L'accent mis par Veil sur une politique européenne en matière de santé publique évoque la communautarisation de nouveaux domaines d'action. Son engagement suggère que les élus avaient la capacité de légitimer, sinon de promouvoir ce processus. Des débats comme celui auquel donna lieu le plan d'action contre le cancer en 1990, indiquent que Veil avait raison lorsqu'elle mettait en

72. Sur la nomination de Veil au poste de ministre de la Santé en 1974, voir M. SZAFRAN, Simone Veil. Destin, op.cit., pp.221-222 et 229; S. VEIL, Une vie, op.cit., p.147.

73. Sur cette stratégie compensatoire, voir W. BEAUWALLET, S. MICHON, op.cit., p.82.

74. Parlement européen, Vos députés: Simone Veil.

75. CARDOC, base de données.

76. O. COSTA, P. MAGNETTE, Idéologies et changement institutionnel dans l'Union européenne. Pourquoi les gouvernements ont-ils constamment renforcé le Parlement européen?, in: Politique européenne, 9(2003), pp.62-64; R. CORBETT, F. JACOBS, M. SHACKLETON, The European Parliament at Fifty: A View from the Inside, in: Journal of Common Market Studies, 2(2003), pp. 360-361. 
avant la capacité du PE de créer un consensus européen sur des problèmes politiques d'actualité. Par leur vote, les députés marquèrent un large accord sur une politique plus ambitieuse que celle que préconisaient les autres instances communautaires.

Veil entra au PE par le jeu des circonstances. Ce tournant dans sa carrière conféra une nouvelle dimension à son profil. Elle qui depuis 1974 incarnait la lutte pour l'émancipation féminine devint une apôtre de la cause européenne. Cette évolution ne fut pas le produit d'un processus de socialisation institutionnelle. Elle résulta plutôt de la conception que se forgea Veil de son rôle de présidente du premier PE élu au suffrage universel direct. Si le mandat européen de Veil influa sur son profil, ellemême eut aussi un impact sur le PE. Par ses rencontres avec des personnalités comme Thatcher et Sadate, la présidente Veil contribua à rehausser le statut du PE. ${ }^{77}$ Elle œuvra en ce sens à un moment où la nouvelle légitimité démocratique du PE nourrissait les espoirs sur son aptitude à donner un nouveau souffle au projet européen. Ce moment serait bref. L'optimisme allait retomber au cours des années quatre-vingt. D'ici là, le $\mathrm{PE}$ aurait néanmoins renforcé sa position face aux autres institutions communautaires, comme en témoigne l'introduction des procédures de consultation et d'avis conforme par l'Acte unique européen de 1985. Veil contribua aussi à faire évoluer le PE en insistant sur son devoir de définir une position et des orientations politiques sur des thèmes comme les droits de l'homme et la santé - l'un des fondements de l'État-providence européen. Le parcours de Veil démontre ainsi la complexité de l'interaction entre institutions et personnalités, susceptibles de s'influencer mutuellement dans un jeu de réciprocité constructive.

77. Sur la venue de Thatcher au PE, voir PE1 P1 214/RECE RECE-19811216 0110, PE, Procès-verbal: invitation de Margaret Thatcher à une période de session, 22.09.1981; PE1 P1 214/RECE RECE-19811216 0100, Veil à Thatcher, 23.09.1981; PE1 P1 214/RECE RECE-19811216 0090, PE, Note concernant la couverture télévisée, 23.11.1981; PE1 P1 214/RECE RECE-19811216 0080, $\mathrm{PE}$, Informations quant au car de reportage pour la session de décembre. 